\title{
eJRIEPS
}

Ejournal de la recherche sur l'intervention en éducation physique et sport

$22 \mid 2011$

Varia

\section{Influence du niveau cognitif sur un apprentissage au choix tactique en dyades symétriques}

Florence Darnis et Lucile Lafont

\section{(2) OpenEdition}

\section{Journals}

Édition électronique

URL : http://journals.openedition.org/ejrieps/4611

DOI : 10.4000/ejrieps.4611

ISSN : 2105-0821

Éditeur

ELLIADD

\section{Référence électronique}

Florence Darnis et Lucile Lafont, «Influence du niveau cognitif sur un apprentissage au choix tactique en dyades symétriques », eJRIEPS [En ligne], 22 | 2011, mis en ligne le 01 janvier 2011, consulté le 27 février 2020. URL : http://journals.openedition.org/ejrieps/4611 ; DOI : 10.4000/ejrieps.4611

\section{(0) (1)}

La revue eJRIEPS est mise à disposition selon les termes de la Creative Commons Attribution 4.0 International License. 
eJRIEPS 22 janvier 2011

Influence du niveau cognitif sur un apprentissage au choix tactique en dyades symétriques

Florence Darnis \& Lucile Lafont

LACES, EA 4140, Université de Bordeaux

Résumé

Cette communication s'appuie sur des travaux de psychologie sociale du développement pour étudier la relation entre le développement cognitif, les interactions sociales et l'apprentissage au choix tactique en sports collectifs. La situation est une montée de balle (2 contre 1 +1) avec des sujets de 11-12 ans, répartis en 4 groupes en fonction de la condition d'apprentissage : avec ou sans interactions verbales, et du niveau de développement opératoire. Les principaux résultats montrent que les sujets bénéficiant de verbalisations en dyades progressent davantage que les sujets sans interactions verbales et que le niveau opératoire influence positivement les progrès réalisés.

Cette étude s'inscrit dans une perspective réflexive et socio-constructiviste de l'apprentissage tactique en sports collectifs (Grehaigne \& Godbout, 1999). Afin de montrer les dimensions cognitives et sociocognitives de l'activité d'apprentissage, nous nous proposons d'étudier l'influence du niveau opératoire et de la verbalisation en dyades sur la capacité à progresser dans une tâche décisionnelle au hand-ball chez des garçons et des filles de 11-12 ans. Pour répondre à cet objectif, seront présentés les résultats d'une analyse quantitative en protocole de quasi expérimentation. Une micro-analyse des dynamiques interactives viendra ensuite compléter ces données.

\section{Introduction}

Dans une situation de jeu, l'élève, plongé dans l'événement, est «bombardé » de stimuli et doit réagir intentionnellement pour tenter de modifier l'évolution de la situation dans un sens favorable pour lui. Le développement de l'intelligence tactique passe donc par la construction de schèmes d'action (Vergnaud, 1996) combinant la reconnaissance des situations de jeu, la disponibilité perceptivo-motrice, la décision et l'effectuation. Le joueur doit sans cesse déployer une intention critique tactique, tout en s'adaptant aux intentions tactiques des autres joueurs. Au plan du développement cognitif, l'avènement au niveau 


\section{eJRIEPS 22 janvier 2011}

de la pensée conceptuelle de ce que Piaget (1950) appelle le combinatoire, aux alentours de 12 ans, va permettre à l'enfant de comprendre comment les différents éléments d'un ensemble s'articulent les uns avec les autres pour lui permettre de fonctionner tout en conservant son équilibre. Les travaux de Menaut (1998) montrent un isomorphisme partiel entre les structures de la pensée formelle et le développement d'une pensée tactique critique. L'acquisition consciente et intentionnelle des différents systèmes de jeu devient dès lors du domaine du possible. Par sa démarche cognitive, le sujet, face à un problème ou une tâche exerce sa réflexion et dégage quelques régularités des actions exercées pour arriver à élaborer des savoirs organisés en systèmes généraux. C'est ce que nous appelons des règles d'action. La formulation de ces règles d'action est un acte fondateur de l'appropriation des savoirs dans l'acte tactique. Rappelons que pour Vergnaud (1996), les règles d'action sont des règles de conduite qui intègrent la prise d'information, l'action au sens strict et le contrôle de l'action. Dans le domaine des habiletés motrices, Grehaigne (1999), définit les règles d'action comme les conditions à respecter et les éléments à prendre en compte pour que l'action soit efficace. Une règle d'action a pour caractéristique d'être consciente, de participer à la planification et à la programmation de l'action en relation avec les pouvoirs moteurs, de participer à l'explication de l'action et enfin de présenter un certain degré de généralisation.

On postule dans cette étude que le niveau de développement cognitif et la verbalisation en situation dyadique sont des facteurs favorisant le développement des compétences tactiques en sport collectif. En effet, l'approche socio-constructiviste centrée sur le conflit socio-cognitif, développée par l'école de Genève (Perret-Clermont, 1979, 1988 ; Doise et Mugny, 1981, 1997) montre le rôle de l'interaction sociale dans le développement cognitif. Ces études mettent en évidence l'importance du conflit de centration dans la structuration individuelle et dans l'élaboration de nouvelles régulations cognitives. L'enfant est amené à coordonner ses actions avec autrui et c'est au travers de ces coordinations qu'il sera en mesure d'élaborer des systèmes d'organisation de ses actions sur le réel. C'est dans ces conditions de coordinations interindividuelles, que l'individu, par un mécanisme de décentration, élaborera ses structures cognitives. En retour, ses progrès cognitifs permettront au sujet de participer à des interactions sociales nouvelles, plus élaborées, qui à leur tour modifieront la structuration de la pensée. Ces travaux s'attachent à explorer comment les processus interactifs participent à l'élaboration même des structures de la connaissance et accusent ainsi un rôle causal dans leur genèse. Cependant l'apparition des progrès n'aurait lieu que si un conflit socio-cognitif (CSC) s'instaure entre les 


\section{eJRIEPS 22 janvier 2011}

partenaires. Le conflit, pour donner lieu à une restructuration cognitive, doit être particulièrement fort et donc, mettre en évidence des désaccords entre les partenaires, désaccords qui doivent être débattus et argumentés afin de donner lieu à un CSC (Doise \& Mugny, 1997). Par ailleurs, Gilly, Fraisse \& Roux, (1988) démontrent l'efficacité possible de modalités d'interaction sans conflit (co-élaboration acquiesçante, co-construction, confrontation avec désaccords non argumentés). Ces travaux mettent en avant l'influence des dynamiques interactives et des mécanismes socio-cognitifs dans la résolution de problèmes. Ils mettent en évidence la supériorité de la co-élaboration sur la résolution de problèmes en situation individuelle, mais également que les divergences de réponses doivent donner lieu à des confrontations pendant la phase de réalisation de la tâche. C'est cette confrontation au moment de la mise en œuvre des procédures qui paraît déterminante. D'autre part, la dynamique interactive ne pourra s'installer et ne sera susceptible d'accroître les compétences du sujet que si la situation problème sollicite une maîtrise individuelle encore insuffisamment installée. Cependant, comme l'a montré Fraysse (1985), l'évolution des comportements sociaux accompagne et se réalise simultanément à l'évolution des comportements cognitifs. L'auteur détermine quatre niveaux de prise en compte d'un partenaire qui se traduisent par quatre comportements interactifs différents. Ainsi, au premier niveau ou niveau de « syntonisation », l'enfant ne semble pas «entendre » les aides verbales de l'expérimentateur. Le deuxième niveau marque l'apparition des premières structures de relation qui implique une décentration suffisante pour modifier plus ou moins sa propre action en référence à l'autre (imitation, conceptualisation très faible). Le troisième niveau, dit de «confrontation » se caractérise par une prise en compte systématique de l'une des propriétés de l'objet et d'une systématisation du rôle du partenaire : le conflit socio-cognitif peut s'établir de manière positive. Au quatrième niveau, dit de coopération, les enfants sont capables de coordonner leurs schèmes pour définir le but à atteindre et déterminer les moyens à mettre en œuvre pour y parvenir. Par ailleurs, l'étude de Fraysse (1992) a mis en évidence le rôle du niveau de développement cognitif dans la capacité à tirer bénéfice des interactions en dyades. Ainsi, selon Fraysse (1992), les modalités d'interaction sont en partie déterminées par le niveau de développement cognitif et en retour agissent sur celuici. La présente étude se situe en continuité avec ces travaux.

Dans le cas de l'acquisition des habiletés motrices, quelques études récentes ont étudié les effets des interactions dyadiques et de coopération. En premier lieu, Arripe-Longueville (d'), Fleurance \& Winnykamen, (1995), montrent la supériorité du travail en situations 


\section{eJRIEPS 22 janvier 2011}

dyadiques symétriques et dissymétriques sur un travail individuel dans l'acquisition d'habiletés motrices complexes individuelles (le salto avant en gymnastique). Au cours d'une étude pilote dans le cas des sports collectifs, Darnis-Paraboschi, Lafont \& Menaut (2002), avaient mis en évidence l'efficacité des interactions dyadiques symétriques sur les choix tactiques lors du post-test différé.

Au plan macroscopique des interactions sociales, Lafont et Winnykamen (1999) situent l'analyse des interactions dans le cadre de la classe et du cours d'EPS, ce qui confère à cette approche une dimension écologique. En s'appuyant sur les travaux de Slavin (1983) et de Johnson et al. (1981) il est montré que les situations de coopération apparaissent plus bénéfiques que celles de compétition et de travail individuel. Dans la même perspective théorique et en sports collectifs, Lafont, Proères \& Vallet (2007) ont mis à l'épreuve expérimentalement les effets de discussions en équipe à propos des stratégies de jeu, sur les relations interpersonnelles et les acquisitions motrices. Cette étude montre que le groupe expérimental, qui est mis en situation de discussion collective à propos des stratégies semble tirer bénéfice des discussions dans les domaines de la participation au jeu et dans celui des compétences tactiques. Pour Bouthier (1988) les verbalisations semblent également constituer des opérations indispensables à l'élaboration, la réalisation et la régulation d'actions motrices collectives. Leur introduction dans le travail de classe devrait permettre de développer une dynamique dans les équipes sportives et entraînerait plus particulièrement des effets de réciprocité entre la contribution individuelle des joueurs et la performance collective. Discussions collectives, verbalisations ont pour vocation de dégager des règles d'action, ou règles de l'action efficace (Grehaigne, 1999), visant à améliorer les connaissances en sports collectifs. Ce travail s'inscrit aussi dans cette perspective car il a pour objet de tester l'influence du niveau de développement logique sur les bénéfices tirés du travail dyadique avec ou sans verbalisation. Pour mesurer cet effet, on a sélectionné plusieurs mesures dépendantes : le projet d'action spécifique à la tâche et les connaissances procédurales mises en œuvre dans l'action lors d'un 2 contre 1 au hand ball. Peu d'études s'intéressent au passage de la connaissance à l'action, de la prise de conscience à la motricité dans le domaine de l'enseignement des sports collectifs. Dans une autre perspective, celle de l'expertise en sport, un courant de recherches anglosaxon envisage les relations entre connaissances déclaratives et connaissances procédurales en sports collectifs. A partir d'entretiens, French et Thomas (1987) montrent que les experts sont davantage capables d'expliquer leur action sous la forme : « si..., alors... » et d'envisager davantage d'alternatives aux situations. Ils donnent de meilleures 


\section{eJRIEPS 22 janvier 2011}

réponses du point de vue des connaissances en jeu et sont davantage capables d'organiser l'information. Pour French et Thomas (1987) connaissances déclaratives et connaissances procédurales vont de pair et pour améliorer la prise de décision et la qualité des inférences de l'ordre du «si...alors ...il faut faire... » il faut développer les connaissances déclaratives du même type.

En continuité avec les travaux de Fraysse (1992), l'hypothèse de cette étude concerne l'existence d'une influence du niveau opératoire sur les progrès réalisés en situation d'interaction dyadique dans le cas d'une tâche décisionnelle en sports collectifs. Ainsi on s'attend à ce que seuls les sujets de niveau opératoire intermédiaire-formel tirent bénéfice du travail en dyade. L'étude de l'effet du genre montre qu'il existe une différence initiale entre les garçons et les filles pour certaines mesures (efficacité de l'action) mais que garçons et filles bénéficient tout autant de la phase d'interaction verbale et les progrès réalisés ne se différencient pas selon le genre, quelque soit le groupe, témoin ou expérimental (Darnis-Paraboschi, Lafont et Menaut, 2005).

Nous formulons l'hypothèse selon laquelle, dans le processus d'apprentissage en sports collectifs, le sujet construit ses connaissances par une activité intra-cognitive et une activité inter-cognitive.

\section{Méthode}

\subsection{Sujets}

La population est issue d'élèves de 3 classes de $6^{\text {ème }}$ d'un collège français de milieu rural. La moyenne d'âge est de 11.2 ans ( $S D=0,42)$. 40 participants, 20 filles et 20 garçons ont été retenus sur 72 élèves au total. Les élèves sont novices dans l'activité et sont volontaires pour participer à cette étude. Ils savent qu'ils vont être filmés mais que leur anonymat sera respecté. Dans un premier temps, chaque participant a effectué une épreuve de raisonnement logique ou ECDL: Echelle Collective de Développement Logique (INOP-CNAM, 1973) basée sur les travaux de Longeot (1969). Cette épreuve collective se déroule dans une salle de classe, les élèves sont regroupés par classe et la passation dure une heure. Dans un second temps, l'acte tactique est évalué dans une situation de montée de balle sur tout le terrain de handball à deux joueurs contre un défenseur dans chaque moitié du terrain ( 2 contre $1+1)$. Pour l'évaluation initiale, les joueurs ont changé de partenaires et d'adversaires afin de mesurer leur niveau initial indépendamment du contexte de coopération et d'opposition. Des dyades ont pu ainsi être constituées par genre, symétriques du double point de vue du niveau de développement 


\section{eJRIEPS 22 janvier 2011}

logique et du niveau initial dans la tâche. 20 dyades symétriques ont ainsi été formées, 10 dyades féminines et 10 dyades masculines. 10 dyades ( 5 dyades $F$. +5 dyades $G$.) ont composé le groupe expérimental et 10 dyades ( 5 dyades $F$. +5 dyades $G$.) ont composé le groupe témoin. Les groupes témoin et expérimental ont été triés de manière aléatoire.

\subsection{Mesures}

L'Echelle Collective de Développement Logique comprend 6 items du stade Concret, 6 items du stade Intermédiaire, 4 items du stade Formel A et 4 items du stade Formel B. Chaque item est codé 1 point, ce qui constitue une échelle de 0 à 20 mesurant l'accès au stade formel. Notre étude portant sur des sujets élèves de classes de Gèmes, nous utiliserons la répartition définie par les auteurs (INOP-CNAM, 1973) pour proposer un classement en 2 groupes, un groupe Concret (scores entre 0 et 8) et un groupe Intermédiaire ou Formel (scores $>8$ ).

Un projet d'action (PA) est mesuré par un questionnaire demandant aux sujets de répondre par écrit à la question suivante: "Dans une situation de 2 attaquants contre 1 défenseur, que faut-il que vous fassiez, à deux, pour arriver à tirer au but à chaque fois sans perdre le ballon? " et "Décrire la ou les possibilités dans cette situation et envisager la ou les solutions. " Les réponses ont été évaluées de la manière suivante : 1 point pour «il faut faire des passes"; 2 points pour «il faut faire des passes et se démarquer du défenseur »; 3 points « il faut faire des passes ou dribbler et se démarquer du défenseur » et 4 points pour : "si le défenseur fait ceci..., alors il faut faire cela... » La mesure du projet d'action correspond donc à une échelle de 0 à 4 .

La pertinence du choix (PC) repose sur une évaluation des prises de décision des attaquants lors de la montée de balle $(2$ contre $1+1)$. Trois enseignants, experts dans l'activité, ont observé les joueurs et comptabilisé pour chacun d'eux les "bons choix» $(+)$; les « choix neutres » (+/-) et les « mauvais choix » (-). Un test de concordance (test de Kendall) a été réalisé et ne montre pas de différence entre les évaluations des trois experts. Une échelle a été testée (Darnis-Paraboschi, Lafont \& Menaut, 2002) et utilisée pour cette recherche. Pour chaque joueur la somme des actions positives (+) est divisée par la somme de toutes les actions (+,-) et multipliée par 20. Cette échelle allant de 0 à 20 correspond donc un rapport des bons choix sur le total des actions. Les actions des défenseurs ne sont prises en compte dans cette mesure. Cependant, le rapport d'opposition est contrôlé puisque chaque dyade attaquante rencontre toujours la même dyade défensive. Ainsi, les progrès réalisés ne sont pas dépendants d'un rapport de force fluctuant. 


\section{eJRIEPS 22 janvier 2011}

Le score de l'efficacité de l'action (EA) correspond à un point par réussite de l'action, c'est à dire lorsque les participants arrivent à la zone et tirent au but. Les sujets ayant cinq tentatives, une échelle de 0 à 5 est ainsi constituée.

\subsection{Procédure}

L'expérimentation comprend un pré-test avec l'évaluation des mesures déclarative (PA), comportementale (PC) et de performance (EA). La phase d'entraînement consiste, pour les dyades expérimentales (Exp), d'alterner trois passages dans la situation motrice de choix tactique $(2$ contre $1+1)$ et trois séquences de verbalisations de trois minutes chacune enregistrées soit avec un camescope (Hitachi $8 \mathrm{~mm}$ ), soit avec un dictaphone (Firstline Micro Cassette Recorder). Les participants sont alors invités à se mettre d'accord verbalement pour envisager la ou les solutions permettant de réussir la situation. Pour les dyades témoins (Tém.), il s'agit d'alterner trois passages dans la situation motrice (2 contre $1+1$ ) et trois séquences de passes à 10 mètres de distance pendant trois minutes. Consigne : faire le maximum de passes, sans faire tomber le ballon en $3 \mathrm{mn}$. Un post-test immédiat identique au pré-test et un post-test différé, une semaine après la phase d'entraînement mesurant PC et EA ont été réalisés. La situation de montée de balle avec opposition est identique entre le pré-test la phase d'entraînement et les post-tests. En effet, afin de mesurer les progrès, les relations de coopération et d'opposition sont restées inchangées.

Afin d'analyser les résultats, les sujets ont été répartis en (2X2) groupes en fonction de la condition d'apprentissage: expérimental, avec verbalisations (Exp) vs témoin, sans verbalisation (Tém) et en fonction du niveau de développement opératoire : concret (C) vs intermédiaire/formel (IF).

- G1 : groupe avec interactions verbales, niveau opératoire intermédiaire ou formel

- G2 : groupe avec interactions verbales, niveau opératoire concret

- G3 : groupe sans interactions verbales, niveau intermédiaire ou formel

- G4 : groupe sans interactions verbales, niveau concret

Une ANOVA a été réalisée au pré-test et au post-test pour mesurer les effets principaux de la condition d'apprentissage et du niveau de raisonnement logique. Une analyse de variance à mesure répétée (RMANOVA) a permis de mettre en évidence les progrès entre pré-test et post-tests.

\section{Résultats}

3. 1. Résultats au pré-test et au post-test 
eJRIEPS 22 janvier 2011

Au niveau du projet d'action, les résultats sont présentés au tableau 1 et figure 1.

Tableau 1. Moyenne et écart type du PA par groupes

\begin{tabular}{|l|l|ll|}
\hline Groupe & \multicolumn{2}{|l|}{ Pré test } & \multicolumn{2}{l|}{ Post test } \\
\hline G1 & $2,3 \quad(0,82)$ & $2,8 \quad(1,03)$ \\
\hline G2 & $1,2 \quad(0,78)$ & $2,2 \quad(0,92)$ \\
\hline G3 & $1,6 \quad(0,74)$ & $2,3 \quad(1,30)$ \\
\hline G4 & $1,9 \quad(1,16)$ & $1,5 \quad(0,90)$ \\
\hline
\end{tabular}

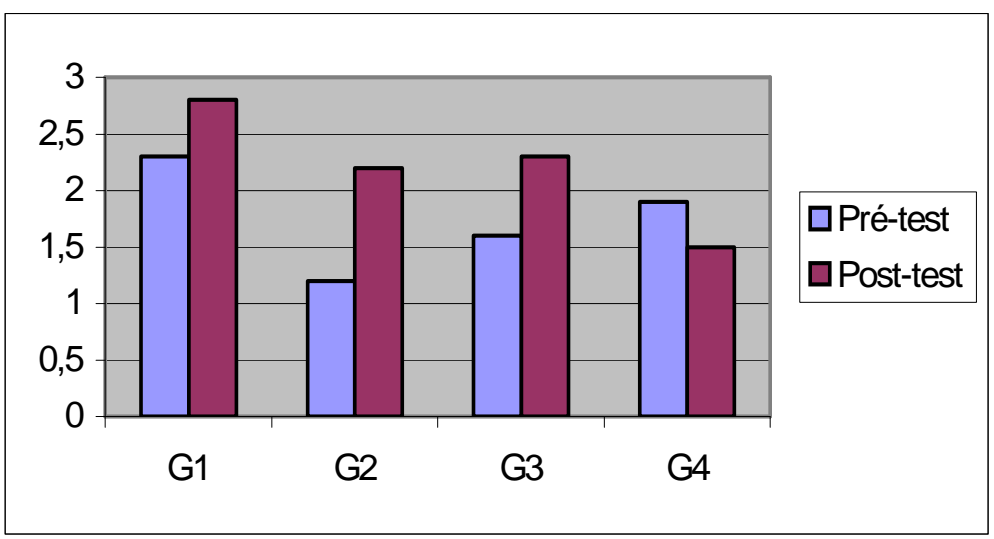

Figure 1. Evolution au PA

Au post-test, l'ANOVA met en évidence un effet principal du niveau opératoire $(F(1,36)=$ $4.50, \mathrm{p}<.05)$ mais pas d'effet de groupe. RMANOVA montre un progrès significatif $(\mathrm{F}(1$, $36)=13.13, p<.001$ ) et un effet d'interaction significatif entre les progrès réalisés et le groupe $(F(3,36)=5.40, p<.01)$. Les comparaisons par groupes mettent en évidence que les groupes «Exp-C », «Exp-IF » et «Tém-IF » progressent significativement (respectivement, $F(1,10)=11.25,5,9, p<.05)$. Les groupes de niveau intermédiaire/formel progressent quelle que soit la condition d'apprentissage. Seul, le groupe témoin de niveau concret ne progresse pas.

Au niveau de la pertinence du choix, les résultats figurent au tableau et figure 2. 
Tableau 2. Moyenne et écart type du PC par groupes

\begin{tabular}{|l|l|l|l|}
\hline Groupe & Pré test & Post test immédiat & Post test différé \\
\hline G1 & 13,1 & 16 & 15,7 \\
\hline G2 & 12,5 & 13,7 & 14 \\
\hline G3 & 12,1 & 13,2 & 13,5 \\
\hline G4 & 12,2 & 12 & 11,5 \\
\hline
\end{tabular}

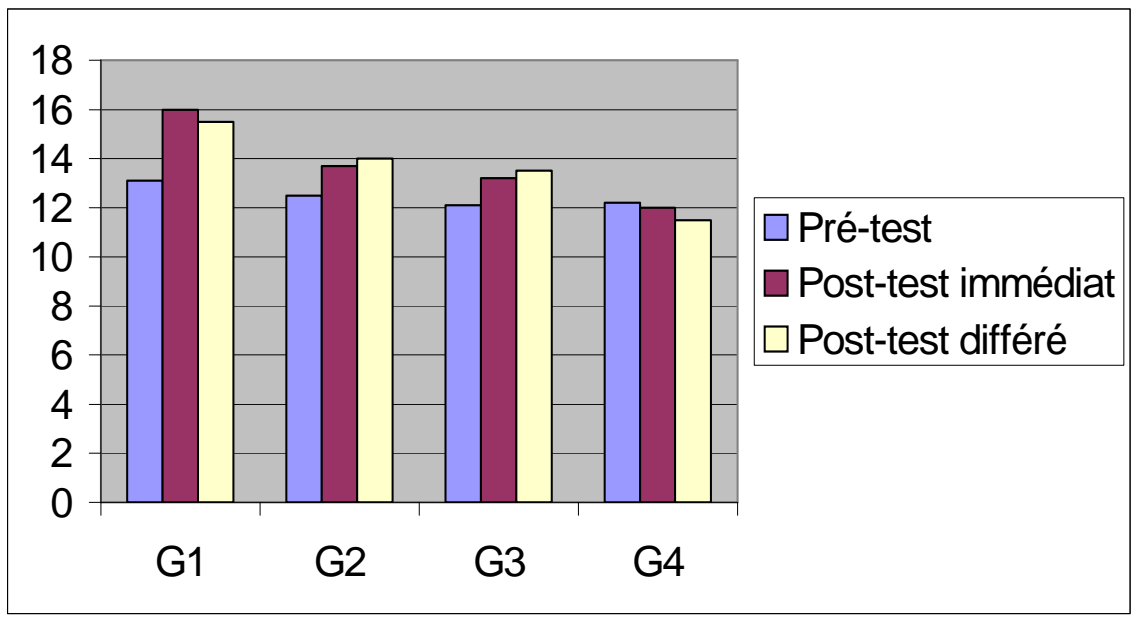

Figure 2 : Evolution au PC

Au post-test immédiat, l'ANOVA ne montre ni d'effet du niveau de développement logique, ni d'effet de groupe (seulement une tendance en faveur des groupes avec interactions dyadiques $(p<.07)$.

Au post-test différé, l'ANOVA montre un effet de groupe $(F(1,36)=5.55, p<.05)$. Les sujets en interactions dyadiques réalisent des choix plus pertinents que ceux qui ne bénéficient pas des interactions. On n'observe pas d'effet significatif du niveau de développement logique (seulement une tendance en faveur des groupes de niveau intermédiaire / formel $(\mathrm{p}=.07)$. RMANOVA montre un progrès significatif au post test immédiat $(F(1,36)=7.32, p<.01)$ et au post test différé $(F(1,36)=18.09, p<.001)$ et une interaction significative entre les progrès et le groupe uniquement lors du post-test différé $(\mathrm{F}(1,36)=7.37, \mathrm{p}<.001$ ) (pour le post-test immédiat, $\mathrm{F}(1,36)=2.27, \mathrm{p}=.09$, ns). Les comparaisons par groupes mettent en évidence plusieurs constats. Au post test immédiat, 


\section{eJRIEPS 22 janvier 2011}

seul le groupe «Exp-IF » progresse significativement $(F(1,10)=11.75, p<.05)$. Au test de rétention, ou post test différé, les groupes «Exp-C», « Exp-IF », « Tém-IF » progressent significativement (respectivement, $F(1,10)=13.5,12.52,8.96, p<.05$ ). Il y a, d'une part un bénéfice plus important lié à la condition d'apprentissage avec verbalisations et, d'autre part, un bénéfice plus important pour les sujets de meilleur niveau opératoire.

Au niveau de l'efficacité de l'action les résultats figurent au tableau 3.

Tableau 3. Moyenne et écart type de l'EA par groupes

\begin{tabular}{|l|l|l|l|}
\hline Groupe & Pré test & Post test immédiat & Post test différé \\
\hline G1 & 2,2 & 3,3 & 3 \\
\hline G2 & 2,8 & 2,9 & 3 \\
\hline G3 & 3 & 3,2 & 3,25 \\
\hline G4 & 3,5 & 4,1 & 3,5 \\
\hline
\end{tabular}

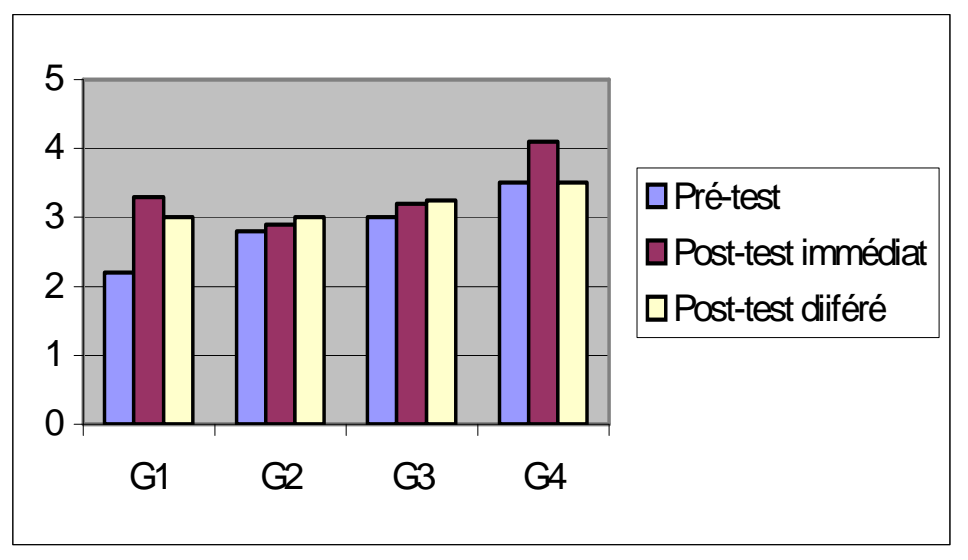

Figure 3. Evolution de l'EA

L'ANOVA ne met en évidence ni effet de groupe ni effet de développement opératoire ni effet d'interaction entre les 2 facteurs. RMANOVA montre un progrès significatif au post test immédiat $(F(1,36)=8.23, p<.01)$ et au post test différé $(F(1,36)=10.84, p<.01)$ mais un effet d'interaction significatif entre le progrès et le groupe uniquement lors du post test différé $(F(1,36)=3.49, p<.05)$. Les comparaisons spécifiques montrent que seul le groupe «Exp-IF » progresse significativement entre le pré test et le post test immédiat et entre le pré test et le post test différé (respectivement, $F(1,10)=37.55,36, p<.05$ ). 


\section{eJRIEPS 22 janvier 2011}

\section{2. Analyse qualitative des interactions verbales}

L'analyse des processus interlocutoires doit permettre de dégager des régularités dans les processus conversationnels et les connaissances mises en jeu dans les interlocutions. II s'agit d'essayer de comprendre dans le cadre d'une micro-analyse des interactions, pour un type de tâche et dans un contexte situationnel, par quels processus le déroulement effectif d'une interaction de co-résolution peut-être bénéfique ou non. Le cadre d'analyse se situe dans la perspective de l'analyse conversationnelle décrit par Gilly, Roux et Trognon (1999). Trognon (1999) définit l'analyse conversationnelle comme une démarche d'investigation des structures normatives des raisonnements impliqués dans la compréhension et la production de l'intelligibilité du déroulement de l'interaction. Elle étudie l'organisation séquentielle de l'interaction et du raisonnement qui l'habite. La conversation est considérée comme une matrice de la pensée et la dynamique de l'interlocution produit conjointement du social et du cognitif. Trognon, St Dizier De Almeida \& Grossen (1999) mettent en évidence le fait que la fonction jouée par le travail en collaboration ne pouvait se décrire comme le résultat de l'addition des ressources mises en œuvre par les partenaires, mais comme une construction conjointe, originale et émergeant de la dynamique interactive. Sur cette base, les auteurs se situant dans une perspective post-piagétienne et post-vygotskienne, indiquent que la cognition en situation interactive, constitue une activité dialogique et située.

\section{2. 1. Contexte situationnel}

La situation de communication décrite ici est impositive. Les participants sont regroupés par groupe classe, dans un lieu identifié comme le lieu du cours d'E.P.S. et la situation motrice rappelle fortement des situations d'apprentissage abordées en classe avec le professeur d'E.P.S. La disposition spatio-temporelle des participants est induite par la position de la fiche sur laquelle figure les consignes et la présente du caméscope ou du magnétophone. La présence du caméscope ou du magnétophone perturbe les interactants. Le temps conversationnel est imposé : trois fois trois minutes. Ce temps est indiqué aux participants et vient parfois interrompre la conversation parfois au moment même où le débat d'idée s'installe, de sorte que la solution proposée par la personne reste en suspend et prend la forme d'un langage intérieur poursuivant son chemin. Le document écrit joue le rôle d'un acte illocutoire de type directif. II a le but de commander une réflexion sur la situation de terrain et sollicite la formulation de règles d'action. 


\section{eJRIEPS 22 janvier 2011}

\section{2. 2. Cadre d'analyse}

L'analyse des interlocutions portera sur la dynamique interactive en reprenant la classification de Gilly, Fraisse et Roux (1988). Ainsi, nous tenterons de dégager quatre types de co-élaborations dans la recherche conjointe de solutions: la co-élaboration acquiescante : un des deux sujets élabore seul une solution, le partenaire ratifie ; la coconstruction : l'action de l'un des sujets est reprise ou poursuivie par l'autre, le premier prenant à nouveau le relais et ainsi de suite; les confrontations avec désaccords correspondant à la présence de désaccords non argumentés; les confrontations contradictoires se définissant par la présence de désaccords argumentés et/ou d'une autre proposition de procédure, dynamique propre au CSC.

\section{1.3 Résultats}

Tableau 4. Répartition des dynamiques interactives dominantes des dyades selon le niveau opératoire des interactants.

\begin{tabular}{|c|c|c|}
\hline Dynamique Interactive & $\begin{array}{l}\text { Dyades de niveau } \\
\text { opératoire concret }\end{array}$ & $\begin{array}{l}\text { Dyades de niveau opératoire } \\
\text { Intermédiaire/Formel }\end{array}$ \\
\hline $\begin{array}{l}\text { Co-élaboration } \\
\text { acquiesçante }\end{array}$ & 2 & 0 \\
\hline Co-construction & 2 & 2 \\
\hline $\begin{array}{l}\text { Confrontation } \\
\text { contradictoire avec } \\
\text { désaccord non argumenté }\end{array}$ & 0 & 2 \\
\hline $\begin{array}{l}\text { Confrontation } \\
\text { contradictoire avec } \\
\text { désaccord argumenté }\end{array}$ & 1 & 1 \\
\hline
\end{tabular}

L'analyse des dynamiques interactives différentielle fait apparaître des modalités interlocutoires variées. II semble que, conformément aux travaux de Fraysse (1992), les sujets plus avancés au plan opératoire manifestent une meilleure prise en compte du partenaire et soient davantage enclins à débattre efficacement pour construire des règles d'action. Ainsi, nous observons une relation entre le niveau de compétence tactique mesuré et la capacité à argumenter un point de vue efficace.

La présente étude se proposait de mettre en évidence le rôle des interactions verbales et du niveau de développement cognitif dans l'apprentissage d'un sport collectif comme le handball. II s'avère que, conformément aux travaux de Arripe-Longueville (D') (1998) les 


\section{eJRIEPS 22 janvier 2011}

situations d'interaction dyadiques sont plus efficaces que les situations sans interaction. Par ailleurs, en accord avec les résultats de Fraysse $(1985,1992)$ concernant la relation entre le développement cognitif et la prise en compte d'un partenaire dans une situation coopérative, les sujets plus avancés sur le plan opératoire tirent d'avantage de bénéfices des apprentissages coopératifs en sports collectifs. Les participants du groupe expérimental, bénéficiant d'interactions verbales entre pairs, progressent davantage que ceux du groupe témoin, n'ayant pas interagi verbalement entre les séquences de jeu. Dans le cas de la pertinence du choix, au post test immédiat et au test de rétention, et du projet d'action, cette expérimentation montre que les sujets d'un niveau de développement logique plus avancé ou bien les sujets moins avancés bénéficiant de verbalisations réalisent des progrès significatifs. Pour ce qui concerne le groupe témoin, les sujets de niveau opératoire Intermédiaire-Formel progressent plus que les sujets de niveau Concret pour le projet d'action et pour la pertinence du choix au test de rétention. De plus, il nous semble intéressant de pointer que dans une situation sans interactions verbales, sans remédiation et sans procédure de guidage, seuls les élèves en avance sur le plan opératoire progressent. Ils semblent capables de réaliser des inférences sur le jeu pour construire, dans un processus intra-cognitif des règles d'action leur permettant de développer des compétences tactiques. Pour les sujets moins avancés sur le plan cognitif l'interaction sociale permet de faire émerger, dans un processus inter-cognitif des règles d'actions qui peuvent être débattues et mises en confrontation avec la situation de jeu. Cependant ces résultats doivent être nuancés à l'éclairage de certaines limites dues à la méthode expérimentale et au type de tâche utilisée. En effet, si les pré-tests ne font pas apparaître de différences de groupe, il n'en demeure pas moins vrai que le nombre limité de sujets et la complexité des variables utilisées, ne nous a permis d'équilibrer parfaitement les groupes lors des pré-tests. Enfin, nous avançons deux facteurs expliquant que les effets significatifs observés dans cette étude puisse sembler relativement circonscrits. En premier lieu, la spécificité des activités sportives collectives, par rapport aux activités individuelles, leur complexité et la présence du rapport d'opposition peuvent limiter les différences. Ensuite malgré le caractère expérimental de notre dispositif, la tâche support et la situation interactive, en respectant les conditions de validité écologique qui sont celles de la leçon d'EPS, ont pu tendre à gommer également certaines différences. 


\section{eJRIEPS 22 janvier 2011}

\section{Conclusion}

Si les aspects cognitifs semblent influencer un apprentissage au choix tactique en sport collectif, la mise en œuvre de modalités socio-cognitives dans l'apprentissage semble porteuse de progrès dans la construction de compétences tactiques. Ces résultats présentent plusieurs intérêts au plan des mises en œuvre didactiques et pédagogiques. En premier lieu, ils permettent de renforcer l'idée selon laquelle un apprentissage en situation interactive est efficace dans le cas d'activités décisionnelles que sont les sports collectifs. Ce constat devrait inciter les intervenants à diversifier les modes de groupements des pratiquants sportifs débutants. Mais nos résultats engagent aussi à considérer que les groupements et les modalités interactives ont une efficacité différentielle selon les pré-requis des interactants. Ce constat renforce l'idée d'une nécessaire variabilité des modes d'intervention et d'un enrichissement de ceux-ci.

Nos travaux se situent dans une perspective socio-constructiviste de l'apprentissage en sports collectifs que nous modélisons de la façon suivante :
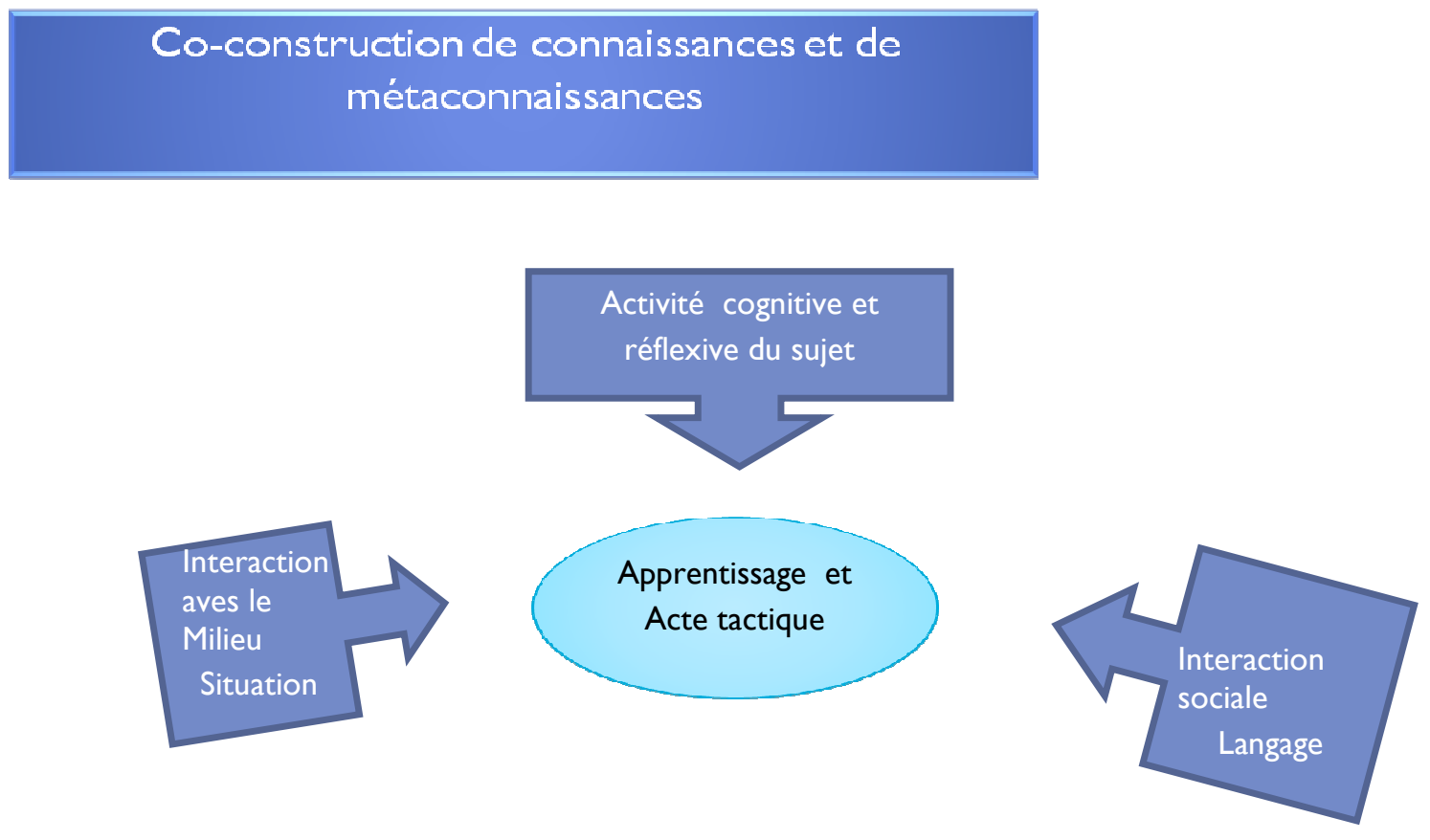

\section{Bibliographie}

Arripe-Longueville, F. (d'), Fleurance, P., \& Winnykamen, F. (1995). Effects of the degree of competence symmetry-assymmetry in the acquisition of a motor skill in a dyad. Journal of Human Movement Studies, 28, 255-273. 


\section{eJRIEPS 22 janvier 2011}

Arripe-Longueville, F. (d') (1998). Contribution à l'identification de processus cognitifs et de mécanismes interactifs impliqués lors de l'acquisition d'une habileté motrice complexe. Thèse en STAPS, Université de Paris X-Nanterre France.

Bouthier, D., «Les conditions cognitives de la formation d'actions sportives collectives », Thèse non publiée, Université de Paris V. 1988.

Darnis-Paraboschi, F., Lafont, L., \& Menaut, A. (2002). Effet des interactions dyadiques sur la compétence tactique en sports collectifs chez des sujets de 11-12 ans. Actes des Journées nationales d'études de la Société Française de Psychologie du Sport, 21-22 mars 2002, Limoges, France, 25-28.

Darnis-Paraboschi, F., Lafont, L., \& Menaut, A. (2005). Influence of dyadic interactions on tactical choices in a collective ball game. European Journal of Psychology of Education, $X X, 2,171-184$.

Doise, W., \& Mugny, G. (1981). Le développement social de l'intelligence. Paris : Interéditions.

Doise, W., Mugny, G. (1997). Psychologie sociale et développement cognitif, Paris: Armand Colin.

Fraysse, J.C. (1985). Relation entre niveau fonctionnel et prise en compte d'un partenaire dans une tâche de tris d'objets. Université de Provence, Archives de Psychologie, 53, 447-452.

Fraysse, J.C. (1992). Relation entre développement cognitif et compétences interactives, Thèse non publiée, H.D.R., Université d'Aix-Marseille.

French, K.E. \& Thomas, J.R. (1987). The relation of knowledge development to children's basketball performance. Journal of sport psychology, 9, p.15-32.

Gilly, M., Fraisse, J.C., \& Roux, J.P. (1988). Résolution de problèmes en dyades et progrès cognitifs chez des enfants de 11 à 13 ans : Dynamiques interactives et mécanismes sociocognitifs. In : A.N. Perret-Clermont \& M. Nicolet (Eds.), Interagir et connaître. Enjeux et régulations sociales dans le développement cognitif. (pp. 73-92). Fribourg : Del Val.

Gilly, M., Roux, J.P., \& Trognon, A. (1999). Apprendre dans l'interaction. Nancy : Presses Universitaires de Nancy.

Grehaigne, J.F. (1999). L'enseignement des sports collectifs à l'école: conception, construction et évaluation, Bruxelles : De Boeck Université.

Grehaigne, J. F., \& Godbout P. (1995). Tactical knowledge in team sports from a socioconstructivist and constructivist perspective, QUEST, 47, 490-505. 


\section{eJRIEPS 22 janvier 2011}

INOP-CNAM (1973). Echelle Collective de Développement Logique, Paris.

Johnson, D.W., Maruyama, G., Johnson, R., Nelson, D., \& Skon, L. (1981). Effects of cooperative, competitive, and individualistic goal structures on achievement : A meta-analysis. Psychological Bulletin, 89, 47-62.

Lafont, L., \& Winnykamen, F. Cooperation and competition in children and adolescents. In Y. Vauden Auweele, F. Bakker, S. Biddle, M. Durand, \& R. Seiler (Eds.), Psychology for educators (pp. 379-404). Champaign, II: Human Kinetics. 1999

Lafont, L., Proères, M. Vallet, C. "Cooperative group learning in a team game : role of verbal exchanges among peers ». Social Psychology of Education, 10, 93-113, 2007.

Longeot, F. (1969). Psychologie différentielle et théorie opératoire de l'intelligence, Paris : Dunod.

Menaut, A. (1998). Le réel et le possible dans la pensée tactique. Contribution à une théorie du jeu sportif. Bordeaux : Presses Universitaires de Bordeaux.

Perret-Clermont, A.N. (1979) La construction de l'intelligence dans l'interaction sociale. Berne : Peter Lang.

Perret-Clermont, A.N., \& Nicolet, M. (1988). Interagir et connaître. Enjeux et régulations sociales dans le développement cognitif. Fribourg : DelVal.

Piaget, J. (1950) Introduction à l'épistémologie génétique, Paris : PUF.

Trognon, A. (1999). Eléments d'analyse interlocutoire. In M. Gilly, J.P. Roux, \& A. Trognon (Eds), Apprendre dans l'interaction, analyse des médiations sémiotiques. (pp. 6994), Nancy : Presses Universitaires de Nancy.

Trognon, A., St Dizier De Almeida, V., \& Grossen, M. (1999). Résolution conjointe d'un problème d'arithmétique, ou comment décrire des cognitions distribuées dans la conversation? In M. Gilly, J.P. Roux, \& A. Trognon (Eds), Apprendre dans l'interaction, analyse des médiations sémiotiques. (pp. 121-141), Nancy : Presses Universitaires de Nancy.

Slavin, R. (1983). When does cooperative learning increase student achievement? Psychological Bulletin, 94(3), 429-445.

Vergnaud, G. (1996). Au fond de l'action, la conceptualisation. In J.M. Barbier (Eds.) Savoirs théoriques et savoirs d'action, (pp. 275-305). Paris : PUF. 